\title{
A Compound Heterozygous HBB: C.79G>A Associated with HBB: C.-78A>G Mutation of HBB Gene Leading to B-Thalassemia Intermedia in A Vietnamese Patient: A Case Study
}

\author{
Nguyen Van Hưng" ${ }^{1 \#}$, Ngo Diem Ngoc ${ }^{2 \#}$, Nguyen Thi Phuong $\mathrm{Mai}^{2}$, Ngo Thị Tuyet Nhung², Lý Thi \\ Thanh $\mathrm{Ha}^{1,2 *}$ and Tien Anh $\mathrm{Ngo}^{3 *}$ \\ ${ }^{1}$ Department of Medical Genetics, Vinmec Health Care System, Vietnam \\ ${ }^{2}$ National Children's Hospital, Vietnam \\ ${ }^{3}$ Vinmec Tissue Bank, Vinmec Health Care System, Vietnam \\ ${ }^{\#}$ These authors have contributed equally to this work as first authors \\ *Corresponding author: Tien Anh Ngo, Vinmec Tissue Bank, Vinmec Health Care System, Vietnam. \\ Lý Thị Thanh Hà, Department of Medical Genetic, Vinmec Health Care System, Vietnam. \\ To Cite This Article: Amal Nguyen Van Hung, Ngo Diem Ngoc, Nguyen Thi Phuong Mai, Ngo Thị Tuyet Nhung, Lý Thi Thanh Ha, Tien Anh Ngo. \\ A Compound Heterozygous HBB: C.79G>A Associated with HBB: C.-78A $>$ G Mutation of HBB Gene Leading to B-Thalassemia Intermedia in A \\ Vietnamese Patient: A Case Study. Am J Biomed Sci \& Res. 2021 - 13(2). AJBSR.MS.ID.001842. DOI: 10.34297/AJBSR.2021.13.001842.
}

Received: 海 May 31, 2021; Published: 監 June 10, 2021

\begin{abstract}
$\beta$-thalassemia is an inherited blood disorder that is the most common single-gene disorder worldwide and in Vietnam. This study reports an intermedia $\beta$ thalassemia patient in a family from Northern Vietnam. The mother has compound heterozygous thalassemia presenting mutation of hemoglobin HBB: c.79G>A associated with HBB: c.-78A $>$ G. The result indicates that the determination of molecular characterization of subjects presenting normal $\mathrm{HbA} 2$ level associated with abnormal red cell indices is needed for accurate diagnosis and improved genetic counseling.
\end{abstract}

Keywords: $\beta$-thalassemia intermediate, HBB: c.79G>A, HBB: c.-78A>G

Abbreviations: Hb: Hemoglobin; MCH: Mean Corpuscular Hemoglobin; MCV: Mean Corpuscular Volume; RBC: Red blood cell count

\section{Introduction}

Thalassemia is an inherited disorder of autosomal disorders with a carrier prevalence of $5 \%$ globally [1]. This disorder is the primary cause of hemolytic anemia worldwide, especially in Southeast Asian countries, including Vietnam. There are two main types of thalassemia, including $\alpha$-thalassemia (causing by mutations in $\alpha$-globin gene) and $\beta$-thalassemia (causing by mutations in $\beta$-globin gene). Clinical severity forms the basis of $\beta$-thalassemia classification, as follows:

a) $\beta$-thalassemia major, the severest type of $\beta$-thalassemia, with patients suffering from severe anemia and transfusion dependency, b) $\beta$-thalassemia intermedia, sporadic or no transfusions are required for anemia,

c) $\beta$-thalassemia minor, called $\beta$-thalassemia trait, this form is usually asymptomatic [2]. This study will focus on the case of $\beta$-thalassemia intermedia without blood transfusion from childhood to the present time.

\section{Materials and Methods}

An unrelated couple in the Vietnamese Kinh group was admitted to the Human Genetics Department, National Children's Hospital, for genetic counseling for thalassemia. They have two children who have been identified as $\beta$-thalassemia major who need treatment 
and regular blood transfusions. Based on laboratory findings, including complete blood count and hemoglobin electrophoresis (Table 1), this couple is highly likely to be $\beta$-thalassemia carriers. DNA testing for mutations in the $H b b$ gene is indicated for both husband and wife to confirm the diagnosis. They are assigned to test for 09 common mutations in the $\beta$-globin gene including: -28 $[A>G]$, codon $17[A>T$ ] , codon 26 [G>A] HbE, IVS 1.1 [G>T], IVS 1.5 $[\mathrm{G}>\mathrm{C}$ ], codon 41/42 [-TTCT], codon 71/72 [+A], codon 95 [+A], IVS $2.654[\mathrm{C}>\mathrm{T}]$ by multiplex ARMS PCR. Sanger sequencing was then performed to confirm. According to the manufacturer, amplification products generated by PCR were purified using PureLink ${ }^{\mathrm{TM}}$ PCR Purification Kit (Invitrogen) and were cycle sequenced using Big Dye v3.1 reagents (Applied Biosystems) protocol. Sequencing products were purified with BigDye XTerminator ${ }^{\mathrm{TM}}$ Purification Kit (Applied Biosystems) and automated sequencing performed by capillary electrophoresis on an ABI3500Dx (Applied Biosystems). Sequences were aligned and examined by using Chromas Pro software (Technelysium Pty Ltd).

Table 1: Hematological parameters.

\begin{tabular}{|c|c|c|}
\hline Parameters & Husband & Wife \\
\hline $\mathrm{Hb}(\mathrm{g} / \mathrm{dL})$ & 140 & 130 \\
\hline $\mathrm{MCV}(\mathrm{fL})$ & 72 & 72.8 \\
\hline $\mathrm{MCH}(\mathrm{pg})$ & 23 & 23 \\
\hline $\mathrm{HbA}_{1}(\%)$ & 94,3 & 35.5 \\
\hline $\mathrm{HbA}_{2}(\%)$ & 5,7 & 0 \\
\hline $\left.\mathrm{HbX}^{(} \%\right)$ & - & HbE: 63.7, HbF: 8.0 \\
\hline Inclusion Bodies & Negative & Negative \\
\hline
\end{tabular}

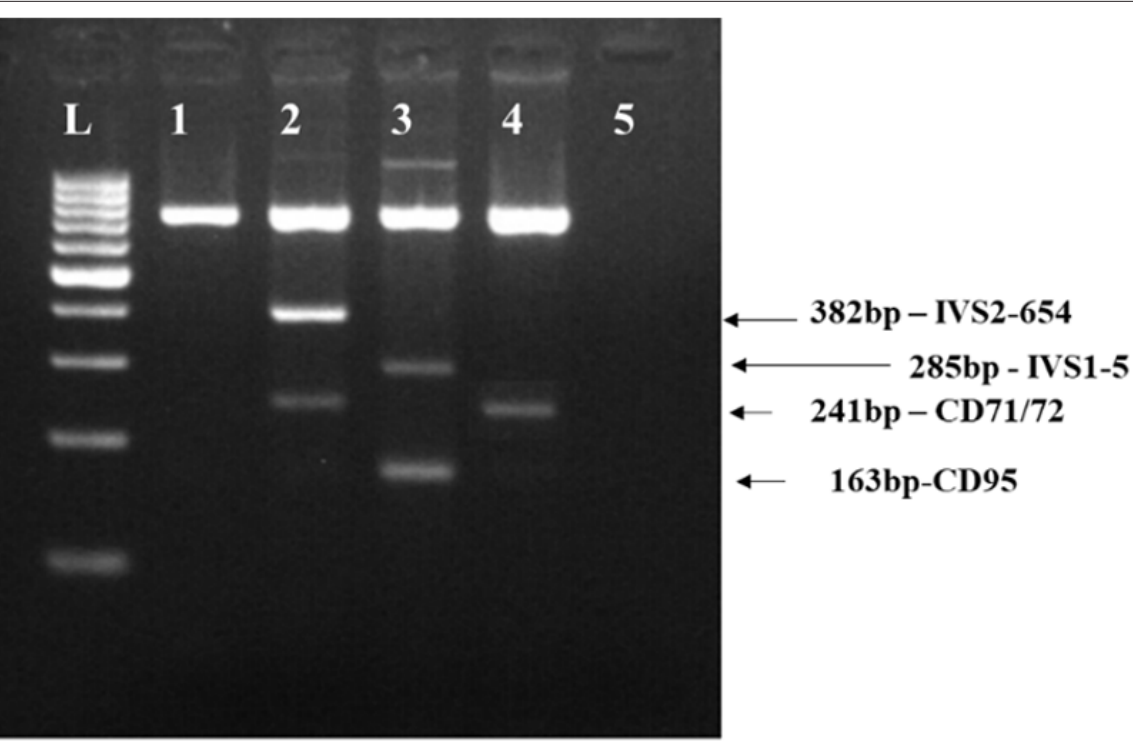

Figure 1: Gel electrophoresis analysis of CD71/72 (+A) mutation in the patient's husband by multiplex ARMS PCR.

Lane L: Ladder 100bp.

lane 1: Negative control.

lane 2: Internal control with IVS2-654(C-T) and CD71/72 (+A) mutant

lane 3: Internal control with IVS1-5 (G-C) and CD95 (+A);

lane 4: Husband's DNA sample.

lane 5: Blank control. 


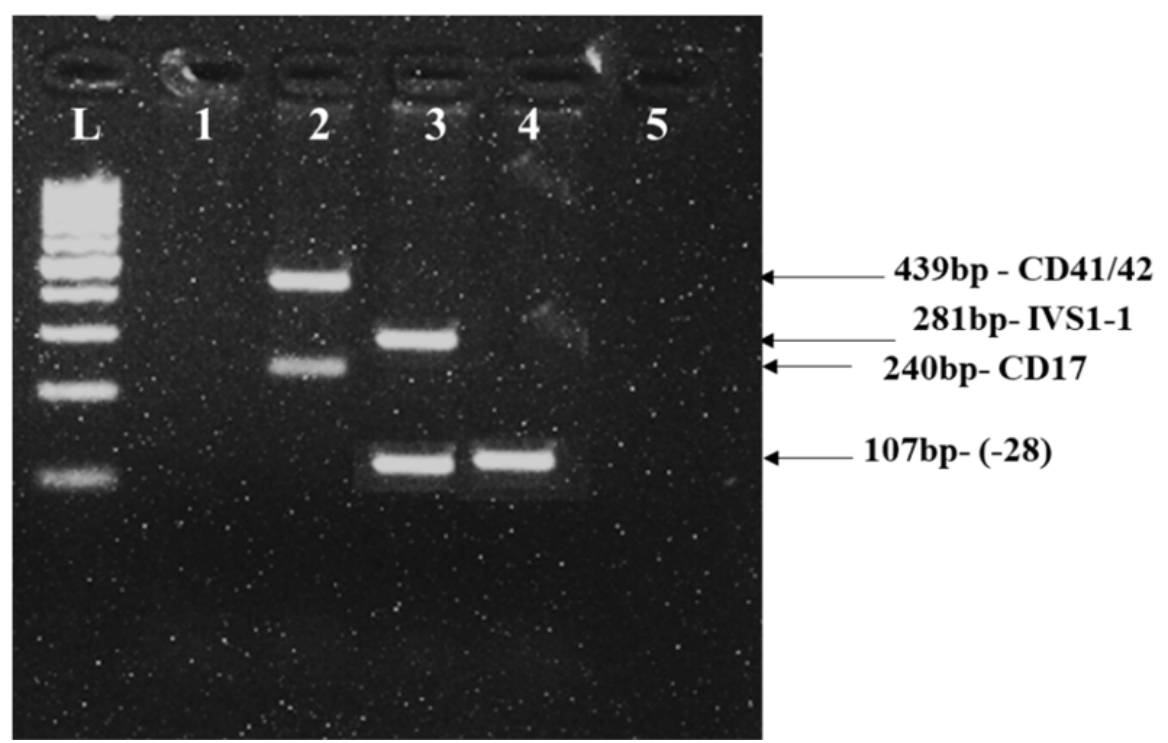

Figure 2: Gel electrophoresis analysis of $-28(\mathrm{~A}-\mathrm{G})$ mutation in the patient's wife by multiplex ARMS PCR. Lane L: Ladder 100bp lane 1: Negative control.

lane 2: Internal control with CD41/42 (-TCTT) and CD17 (A-T) mutant.

lane 3: Internal control with IVS1-1 (G-T) and -28 (A-G).

lane 4: Wife's DNA sample.

lane 5: Blank control.

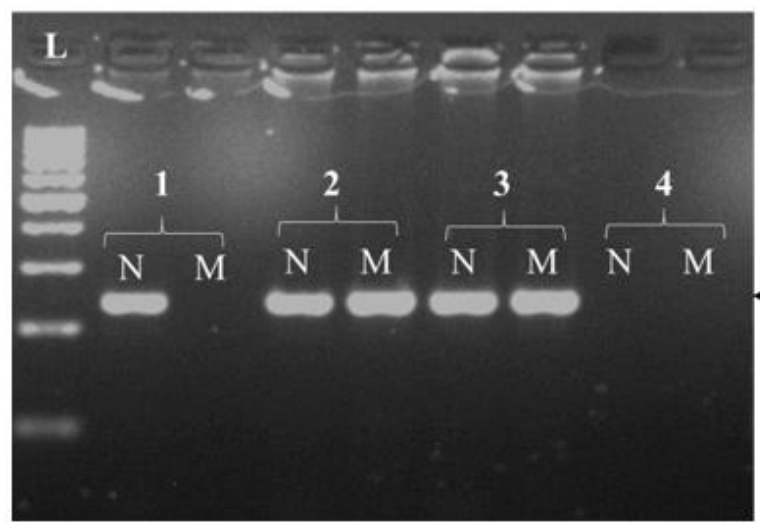

280bp CD26-HBE

Figure 3: Gel electrophoresis analysis of CD26-HbE in the patient's wife by ARMS PCR. Lane L: Ladder 100bp; lane 1: Negative Control lane 2: Internal control with heterozygous $\mathrm{HbE}$.

lane 3: Wife's DNA sample.

lane 4: Blank Control, N: normal band; M: the mutant band.

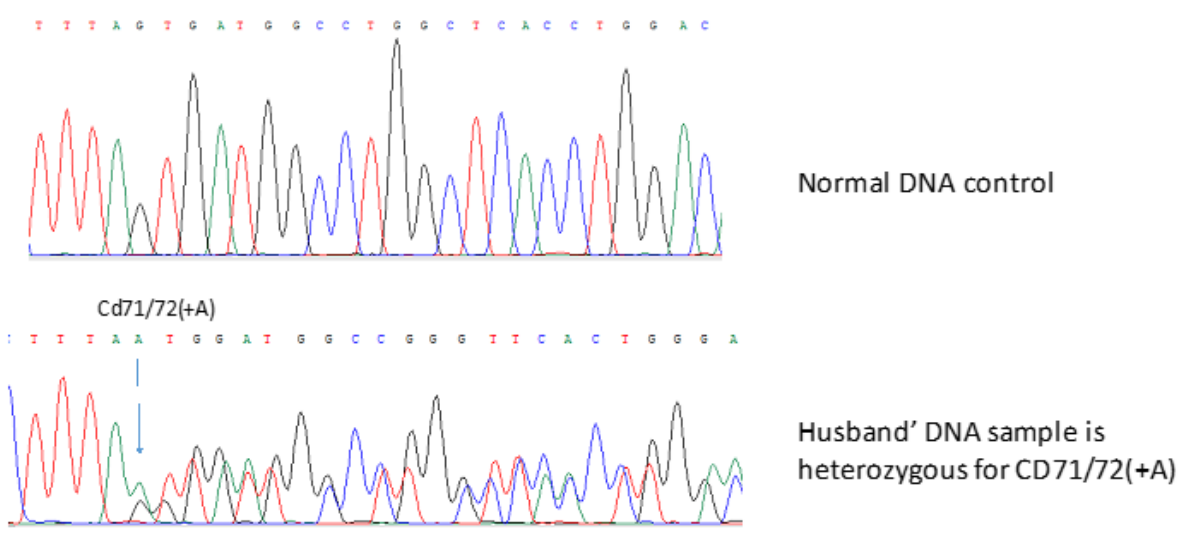

Figure 4: Confirmation of the CD71/72(+A) with husband's DNA sample by Sanger sequencing. 


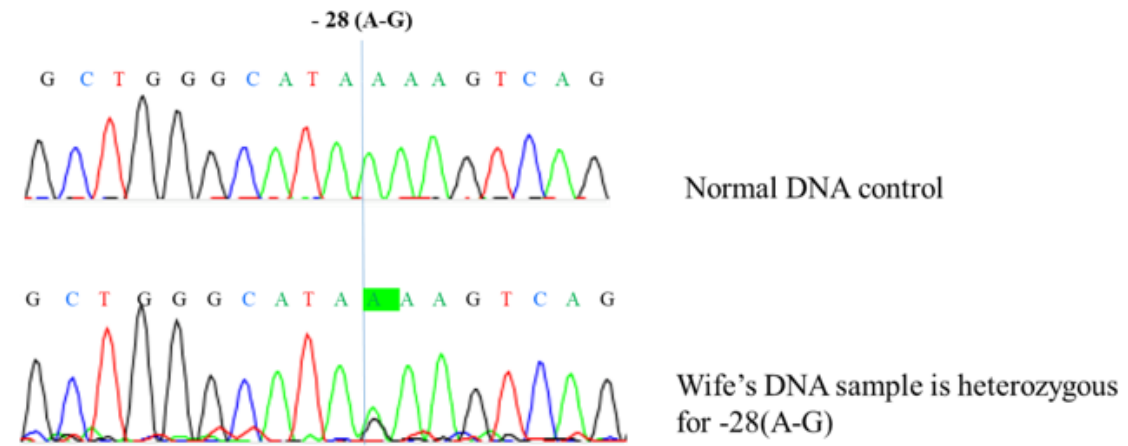

Figure 5: Confirmation of the -28 (A-G) with wife's DNA sample by Sanger sequencing.
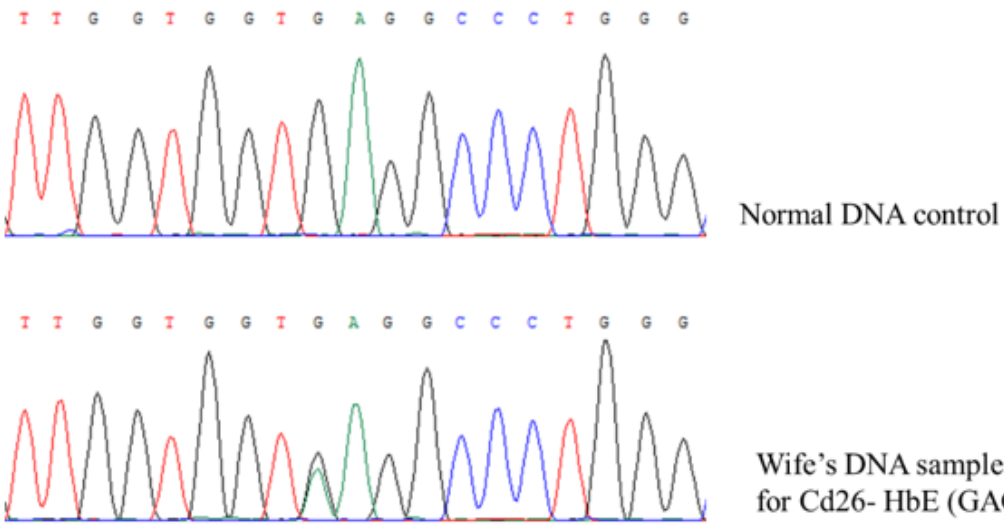

Wife's DNA sample is heterozygous for Cd26- $\mathrm{HbE}$ (GAG-AAG)

Figure 6: Confirmation of the HbE with wife's DNA sample by Sanger sequencing.

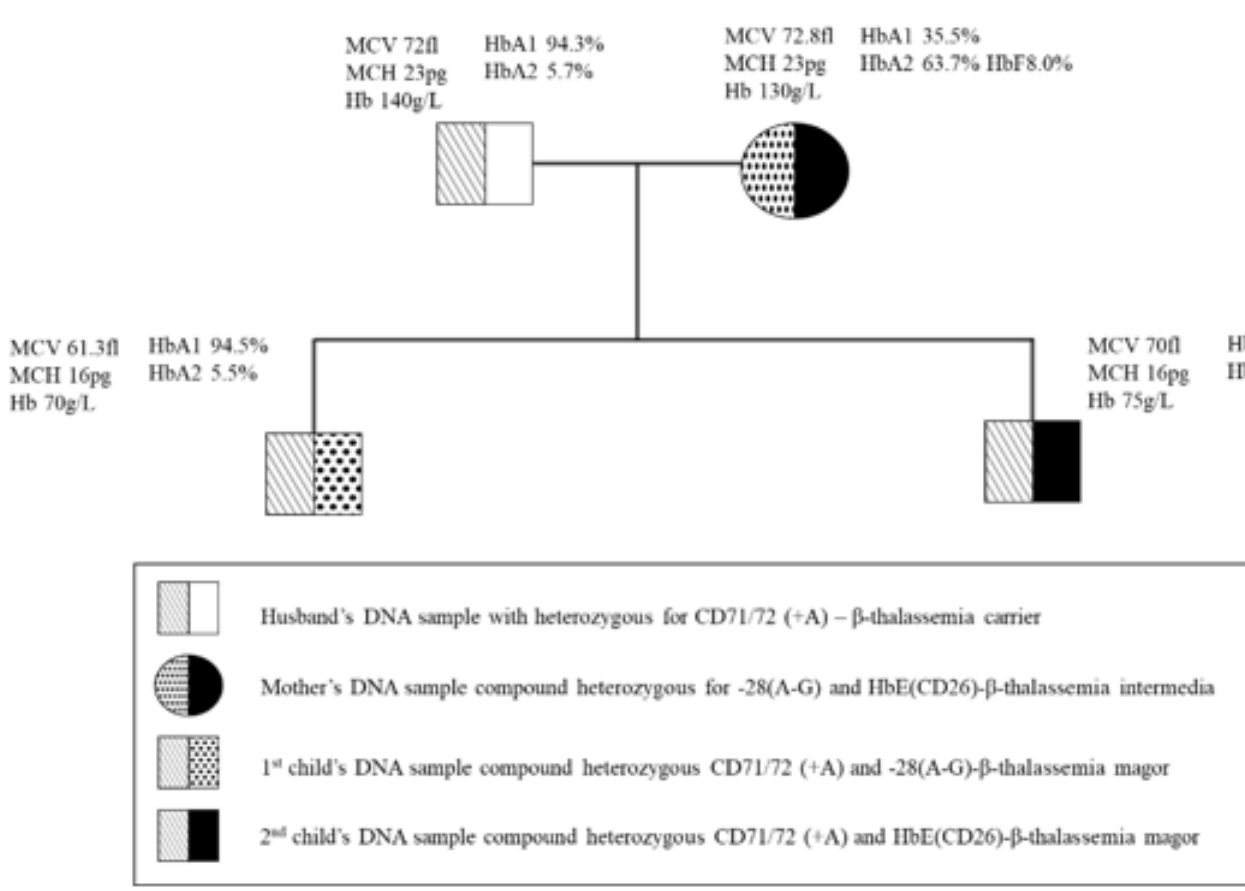

Figure 7: A pedigree chart showing the genotypes of family members. 


\section{Conclusion}

Our data highlight the necessity of deep molecular characterization of intermedia thalassemia subjects. This study plays a significant role in genetic counseling, diagnosis, and treatment, especially in prenatal diagnosis for risk families.

\section{Acknowledgments}

The authors express our sincere to all staff of the Human Genetics Department - Vietnam National Children's Hospital, Medical Genetic Department - Vinmec Health Care System, Vietnam, and Vinmec Tissue Bank.

\section{Conflict of Interest}

The authors declare no conflicts of interest.

\section{References}

1. B Modell and M Darlison (2008) Global epidemiology of haemoglobin disorders and derived service indicators. Bull World Health Organ 86(6): 480-487.

2. V Viprakasit and S Ekwattanakit (2018) Clinical Classification, Screening and Diagnosis for Thalassemia," Hematol Oncol Clin North Am 32(2): 193-211.

3. RM Shawky and TM Kamal (2012) Thalassemia intermedia: An overview Egypt. Egyptian Journal of Medical Human Genetics 13(3): 245-255.

4. K Tari, P Valizadeh Ardalan, M Abbaszadehdibavar, A Atashi, A Jalili, et al. (2018) Thalassemia an update: molecular basis, clinical features and treatment. International Journal Biomedicine and Public Health 1(1): 4858. 